\title{
Laser-plasma interactions in long-scale-length plasmas under direct-drive National Ignition Facility conditions*
}

\author{
S. P. Regan, ${ }^{\dagger, a)}$ D. K. Bradley, A. V. Chirokikh, R. S. Craxton, D. D. Meyerhofer, \\ W. Seka, R. W. Short, A. Simon, R. P. J. Town, and B. Yaakobi \\ Laboratory for Laser Energetics, University of Rochester, 250 East River Road, Rochester, New York 14623- \\ 1299 \\ J. J. Carroll III and R. P. Drake \\ Atmospheric, Oceanic, and Space Sciences, University of Michigan, 2455 Hayword, Ann Arbor, \\ Michigan 48109
}

(Received 19 November 1998; accepted 15 January 1999)

\begin{abstract}
Laser-plasma interaction experiments have been carried out on the OMEGA laser system [T. R. Boehly et al., Opt. Commun. 133, 495 (1997)] under plasma conditions representative of the peak of a 1.5 MJ direct-drive laser pulse proposed for the National Ignition Facility (NIF). Plasmas have been formed by exploding $18-20 \mu \mathrm{m}$ thick $\mathrm{CH}$ foils and by irradiating solid $\mathrm{CH}$ targets from one side, using up to $20 \mathrm{~kJ}$ of laser energy with phase plates installed on all beams. These plasmas and the NIF plasmas are predicted to have electron temperatures of $4 \mathrm{keV}$ and density scale lengths close to $0.75 \mathrm{~mm}$ at the peak of the laser pulse. The electron temperature and density of the exploding-foil plasmas have been diagnosed using time-resolved x-ray spectroscopy and stimulated Raman scattering, respectively, and are consistent with predictions of the two-dimensional Eulerian hydrodynamics code SAGE [R. S. Craxton and R. L. McCrory, J. Appl. Phys. 56, 108 (1984)]. When the solid-target or exploding-foil plasmas were irradiated with an $f / 6$ interaction beam at $1.5 \times 10^{15} \mathrm{~W} / \mathrm{cm}^{2}$, well above the NIF $f / 8$ cluster intensity of $\sim 2 \times 10^{14} \mathrm{~W} / \mathrm{cm}^{2}$, stimulated Brillouin backscattering (SBS) was found to be completely inhibited. A conservative upper limit of direct-backscattered SRS was found to be $\sim 5 \%$ from the solid targets. SRS and SBS are thus unlikely to have a significant impact on target performance at the peak of the NIF direct-drive laser pulse. (C) 1999 American Institute of Physics. [S1070-664X(99)93405-5]
\end{abstract}

\section{INTRODUCTION}

The National Ignition Facility (NIF), currently under construction with a nominal laser energy of $1.8 \mathrm{MJ}$, is expected to achieve ignition in both direct- ${ }^{1,2}$ and indirect-drive $\mathrm{e}^{3}$ configurations. The mission of the University of Rochester's Laboratory for Laser Energetics is to study the direct-drive approach to inertial confinement fusion (ICF), where the capsule is directly irradiated by a large number of symmetrically arranged laser beams. To validate the performance of high-gain, direct-drive target designs planned for the $\mathrm{NIF}^{2}$ an understanding of the laser-plasma interactions in the coronal plasmas of these targets is essential. These interactions include stimulated Raman scattering (SRS), stimulated Brillouin scattering (SBS), the twoplasmon decay instability (TPD), and filamentation. ${ }^{4}$ Their significance for direct-drive capsule performance arises either from detrimental suprathermal-electron generation due to plasma wave-breaking or other nonlinear processes (SRS, TPD), or through a reduction in drive power or drive uniformity (SBS, filamentation).

When the incident laser beam intensity exceeds the threshold levels for the various parametric instabilities,

*Paper B1I2.5 Bull. Am. Phys. Soc. 43, 1639 (1998).

${ }^{\dagger}$ Invited speaker.

${ }^{a)}$ Electronic mail: sreg@1le.rochester.edu power is transferred from the incident laser light to lowerfrequency electromagnetic, electron-plasma, or ion-acoustic waves if the energy $\left(\omega_{0}=\omega_{1}+\omega_{2}\right)$ and momentum conservation $\left(\underline{k}_{0}=\underline{k}_{1}+\underline{k}_{2}\right)$ relationships are satisfied (phase matching). Here, $\omega_{0}$ and $\underline{k}_{0}$ represent the pump-wave (laser) frequency and wave vector. The SRS decay products are an electron-plasma wave and a scattered electromagnetic wave, which are denoted with the subscripts 1 and 2. The TPD results when the incident laser light decays into two electronplasma waves. Phase matching occurs for SRS and the TPD at electron densities $n_{e}$ less than or equal to the quartercritical density $n_{c} / 4$. When the phase-matching conditions are satisfied for SBS, the incident laser light decays into an ion-acoustic wave and a scattered electromagnetic wave.

A 1.5 MJ, $\alpha=3$ ( $\alpha$ is defined as the ratio of the cold fuel pressure to the Fermi-degenerate pressure) laser pulse is planned for an all-DT direct-drive target design on the NIF. ${ }^{2}$ This pulse has a peak-on-target intensity $I_{\text {total }}$ of 2 $\times 10^{15} \mathrm{~W} / \mathrm{cm}^{2}$ (summed over all beams) and a foot intensity of $4 \times 10^{13} \mathrm{~W} / \mathrm{cm}^{2}$. The cluster intensities $I_{\text {cluster }}$ (summed over four individual beams) are approximately a factor of 10 lower than $I_{\text {total }}$. The coronal plasma predicted for this design has an electron temperature $T_{e}$ of $\sim 4 \mathrm{keV}$, a density scale length $L_{n}=\left(n_{e} / \nabla n_{e}\right)$ of $\sim 0.75 \mathrm{~mm}$, and a velocity gradient scale length $L_{v}\left(=c_{s} / \nabla v\right.$, where $c_{s}$ is the ion 
acoustic velocity and $v$ is the velocity) of $\sim 0.5 \mathrm{~mm}$ at the peak of the laser pulse and at densities of $0.1 n_{c}$ to $0.2 n_{c}$. While parametric instabilities have been studied extensively under conditions relevant to indirect-drive ICF, ${ }^{5,6}$ the results presented here represent their first study in NIF-scale directdrive plasmas.

This paper reports on experiments under plasma conditions representative of the peak of the NIF direct-drive laser pulse, since they are the most challenging to create. The experiments were performed on the $30 \mathrm{~kJ}, 351 \mathrm{~nm}, 60$ beam OMEGA laser system, ${ }^{7}$ with distributed phase plates (DPPs) ${ }^{8}$ and $f / 6$ focusing lenses on all beams. The targets, all made of $\mathrm{CH}$, included exploding foils and solids. The exploding foils produced large, mm-scale plasmas with $T_{e} \sim 4 \mathrm{keV}$ and a peak on-axis $n_{e}$ between $0.1 n_{c}$ and $0.2 n_{c}$. These temperatures and densities were diagnosed using time-resolved x-ray and visible (SRS backscattering) spectroscopy, respectively, and the observations were found to be consistent with the predictions of the two-dimensional Eulerian hydrodynamics code SAGE. ${ }^{9}$ The solid-target plasmas were predicted to reach similar temperatures and to have similar density profiles below $\sim 0.1 n_{c}$; however, while they lacked the large plateau region characteristic of exploding foils, they included a critical density surface and were in this sense more representative of NIF conditions.

When both foil and solid-target plasmas were irradiated with an interaction beam at $1.5 \times 10^{15} \mathrm{~W} / \mathrm{cm}^{2}$, the directbackscattered SBS signal was found to be completely absent. Some direct-backscattered SRS was observed in the solidtarget plasmas at a very low level, with a conservative upper limit of $\sim 5 \%$.

Simulations of the NIF direct-drive plasmas were carried out with the one-dimensional hydrodynamics code LILAC ${ }^{10}$ and are presented in Sec. II. The production of the longscale-length plasmas on OMEGA is discussed in Sec. III together with SAGE simulations. Section IV presents the characterization of the plasma electron temperature and density and comparisons with SAGE predictions. The SRS and SBS measurements are presented in Sec. V. The main conclusion of this research (Sec. VI) is that SRS and SBS are not likely to have a significant impact on target performance at the peak of the NIF direct-drive laser pulse.

\section{NIF DIRECT-DRIVE PLASMAS}

LILAC calculations of the $1.5 \mathrm{MJ}, \alpha=3$ direct-drive target are shown in Fig. 1. This figure gives the predicted trajectories of the imploding target, the critical-density surface, and the $n_{c} / 4$ and $n_{c} / 10$ surfaces. It is seen that the density scale length and electron temperature increase with time as the laser pulse makes the transition from the foot to the peak intensity. The dashed line shows the laser power history.

A summary of parameters for the foot and peak of this pulse, including the predicted intensity thresholds $I_{\text {threshold }}$ for the SBS, SRS, and the TPD instabilities, is given in Table I. Approximations for these thresholds were taken from Kruer. ${ }^{4}$ These thresholds may be compared with the cluster intensity $I_{\text {cluster }}$, i.e., the intensity summed over the four beams in a NIF cluster, and the total overlapped intensity $I_{\text {total }}$. In the

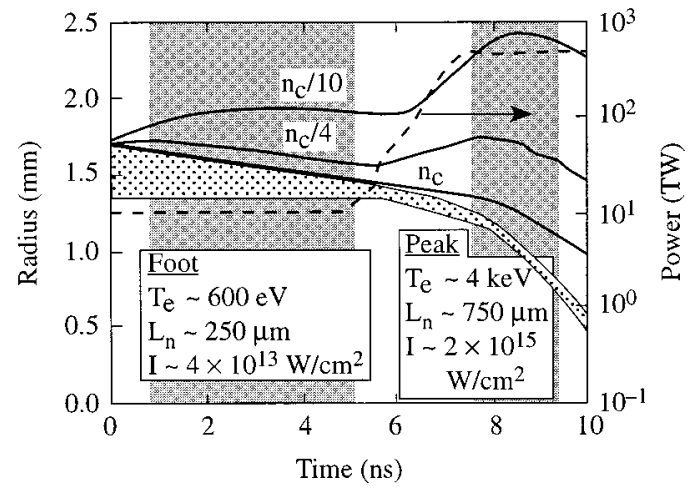

FIG. 1. Radius vs time calculated by LILAC for a high-gain, direct-drive target planned for the NIF with 1.5 MJ and $\alpha=3$. The dotted area represents the solid density region, and the solid lines give the trajectories of the critical, quarter-critical and tenth-critical surfaces. The dashed line indicates the laser power as a function of time. The cross-hatched areas represent the "foot"' and "peak', portions of the pulse, for which the calculated values of electron temperature $T_{e}$, density scale length $L_{n}\left(=n_{e} / \nabla n_{e}\right)$, and on-target intensity $I$ (summed over all beams) are indicated.

case of SRS it is anticipated that the phase-matching conditions will be satisfied only for the light within the $f / 8 \mathrm{NIF}$ cluster, so that the appropriate comparison is with $I_{\text {cluster }}$. In the case of SBS, more than one cluster may drive the instability, but the relevant threshold intensity is likely to be significantly less than $I_{\text {total }}$. As shown in Table I, $I_{\text {cluster }}$ is less than $I_{\text {threshold }}$ for all the instabilities during the foot of the laser pulse. During the peak of the pulse $I_{\text {cluster }}$ is about an order of magnitude lower than $I_{\text {threshold }}$ for SRS and SBS. These comparisons support the expectation that significant SRS and SBS will not occur in direct-drive NIF plasmas. However, the intensities quoted here represent averages over many speckles produced by the phase plates; inside the speckles the peak intensities may be several times higher. It should also be noted that $I_{\text {cluster }}$ is 2.5 times greater than $I_{\text {threshold }}$ for the TPD instability during the peak of the pulse; thus, the TPD instability (not included in the present work because the geometrical configuration is not optimum for its study) needs to be investigated.

\section{PRODUCTION OF LONG-SCALE-LENGTH PLASMAS ON OMEGA}

The long-scale-length plasmas created on OMEGA made use of a design similar to that used on the former

TABLE I. Parameters for the foot and peak portions of the $1.5 \mathrm{MJ}, \alpha=3$, direct-drive target planned for NIF, including coronal plasma conditions and intensity threshold approximations for parametric instabilities.

\begin{tabular}{lcc}
\hline \hline & Foot & Peak \\
\hline$T_{e}$ & $0.6 \mathrm{keV}$ & $4 \mathrm{keV}$ \\
$L_{n}$ & $0.25 \mathrm{~mm}$ & $0.75 \mathrm{~mm}$ \\
$L_{v}$ & $0.5 \mathrm{~mm}$ & $0.5-1.0 \mathrm{~mm}$ \\
$I_{\text {total }}$ & $4 \times 10^{13} \mathrm{~W} / \mathrm{cm}^{2}$ & $2 \times 10^{15} \mathrm{~W} / \mathrm{cm}^{2}$ \\
$I_{\text {cluster }}$ & $4 \times 10^{12} \mathrm{~W} / \mathrm{cm}^{2}$ & $2 \times 10^{14} \mathrm{~W} / \mathrm{cm}^{2}$ \\
$I_{\text {threshold }}$ (SBS) & $4.6 \times 10^{14} \mathrm{~W} / \mathrm{cm}^{2}$ at $n_{c} / 10$ & $2 \times 10^{15} \mathrm{~W} / \mathrm{cm}^{2}$ at $n_{c} / 10$ \\
$I_{\text {threshold }}$ (SRS) & $5.0 \times 10^{15} \mathrm{~W} / \mathrm{cm}^{2}$ & $1.7 \times 10^{15} \mathrm{~W} / \mathrm{cm}^{2}$ \\
$I_{\text {threshold }}$ (TPD) & $3.4 \times 10^{13} \mathrm{~W} / \mathrm{cm}^{2}$ & $8 \times 10^{13} \mathrm{~W} / \mathrm{cm}^{2}$ \\
\hline \hline
\end{tabular}




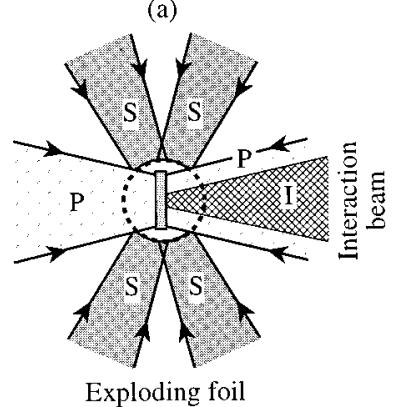

(b)

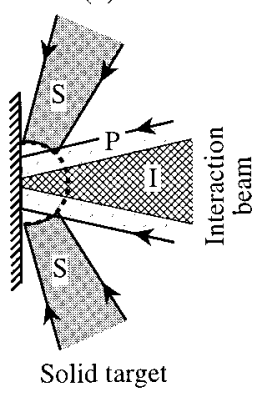

FIG. 2. Geometry for the formation of long-scale-length plasmas on the OMEGA laser system. (a) A CH foil is irradiated and exploded with primary $(P)$ and (delayed) secondary $(S)$ laser beams. (b) A solid CH target is irradiated with primary and secondary laser beams from one side. In both cases, the plasma (shown schematically by the dashed curves) is irradiated by an interaction beam $(I)$ with variable timing.

24-beam OMEGA laser system. ${ }^{11}$ The 60 OMEGA beams were divided into various groups that irradiated the target from different angles and at different times (see Figs. 2 and 3 ). Both exploding foils and solid targets were irradiated first by primary beams $(P)$, whose purpose is to form the plasma, and then by secondary beams $(S)$, which heat the plasma and can be used to control its temperature. The $\mathrm{CH}$ foil targets were $1.2 \mathrm{~mm}$ in diameter, a little larger than the laser spot diameter, and 18-20 $\mu \mathrm{m}$ thick. The solid targets included 1.5-mm-diam planar slabs and partial spheres with thicknesses greater than $80 \mu \mathrm{m}$, predicted to result in similar plasma conditions. In both cases, the plasmas were irradiated by an interaction beam of variable timing, incident along the initial target normal.

The timing sequence of the various groups of beams was similar for foils and solid targets. It is shown in Fig. 3, which gives the time history of the incident, absorbed, and transmitted laser powers. All beams were $1 \mathrm{~ns}$ square pulses with various delays. The primary beams were split into two groups: $P_{1}$, from 0 to $1 \mathrm{~ns}$, with angle of incidence $\sim 20^{\circ}$, and $P_{2}$, from 1 to $2 \mathrm{~ns}$ and incident at $\sim 40^{\circ}$. For the foil targets, these beams served to explode the foils. By the end of the second group $\left(P_{2}\right)$ the foils became underdense on axis, because the phase-plate focal spot distribution is Gaussian-like with its maximum in the center. This resulted in some transmitted laser power starting at $1.5 \mathrm{~ns}$, as seen in Fig. 3(a). The secondary beams arrived from 2 to $3 \mathrm{~ns}$. Their absorption fraction is typically high and they are effective at heating a plasma whose volume is $\sim 1 \mathrm{~mm}^{3}$. For the exploding foils, the transmitted power increases later in time as the plasma expands. The interaction beam typically provides little heating to the plasma and little perturbation to the hydrodynamics, the exception being the solid target after the end of the secondary beams when the interaction beam, if still present, is strongly absorbed and provides some localized heating.

The on-target laser energy was typically $500 \mathrm{~J}$ per beam. The exploding-foil plasmas were irradiated with a total of 19 $\mathrm{kJ}$ of laser energy from 38 beams. Depending on the number of secondary beams used, the solid targets were irradiated with either 19 or 29 beams from one side to deliver $8-12 \mathrm{~kJ}$,
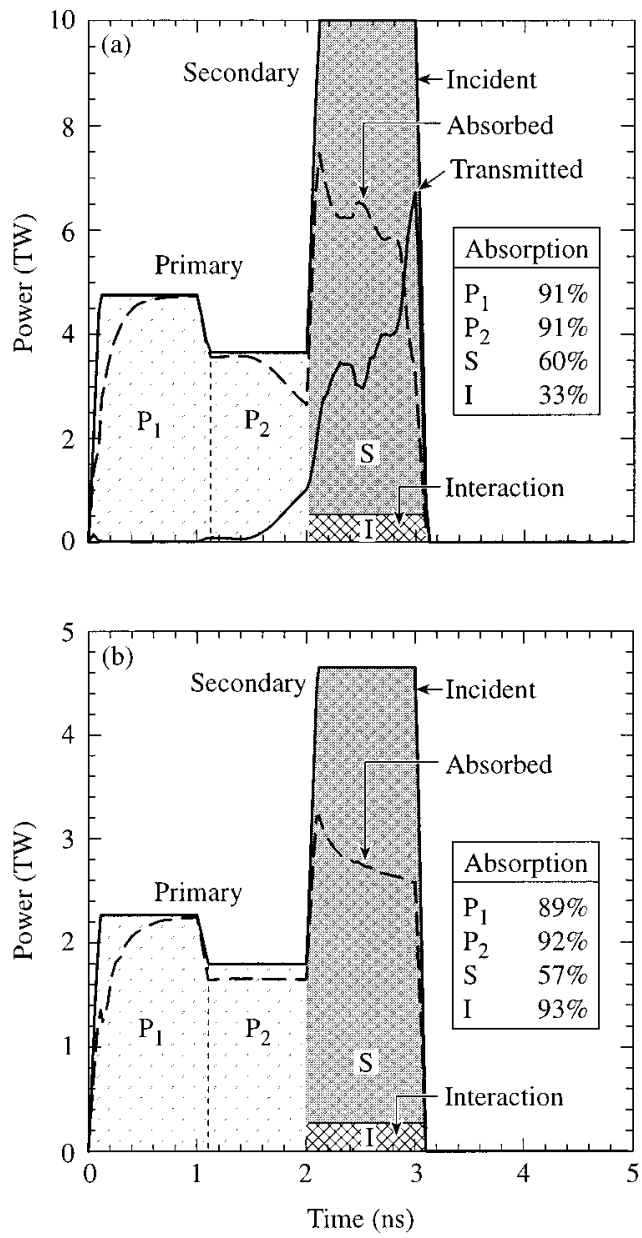

FIG. 3. Timing sequence and total power delivered to the target for $1 \mathrm{~ns}$ flat-top laser pulses used to irradiate (a) exploding foils and (b) solid targets. The interaction beam is fired to probe the NIF-relevant plasma conditions, which usually occur between 2 and $3 \mathrm{~ns}$, and can be timed to start anywhere from 2 to $3 \mathrm{~ns}$. The figure also shows the calculated absorbed and transmitted powers as functions of time, summed over all beams, and the timeintegrated absorption fractions of the primary $\left(P_{1}, P_{2}\right)$, secondary $(S)$, and interaction (I) beams.

producing plasmas with predicted electron temperatures of 3 and $4 \mathrm{keV}$, respectively.

The DPPs used on all beams except the interaction beam produced a focal-spot intensity distribution whose envelope was approximately Gaussian in shape with a full width half maximum diameter of $480 \mu \mathrm{m}$ and a diameter of $950 \mu \mathrm{m}$ enclosing $95 \%$ of the energy. The interaction beam used a different DPP, designed to produce a similar focal-spot shape but scaled down a factor of 2.8 in diameter. The peak intensity in space and time for a nominal $500 \mathrm{~J}$ beam was 1.6 $\times 10^{15} \mathrm{~W} / \mathrm{cm}^{2}$ for the interaction beam and $2.1 \times 10^{14}$ $\mathrm{W} / \mathrm{cm}^{2}$ for the other beams. On most shots $2 \mathrm{D} \mathrm{SSD}{ }^{12}$ was used (on all beams), with a bandwidth of $0.25 \mathrm{THz}$ in the UV.

Contour plots of the predicted $T_{e}$ and $n_{e}$ at $2.6 \mathrm{~ns}$ are shown in Fig. 4 for the two types of plasma. Below eighthcritical density, the two plasmas have very similar density, temperature, and velocity profiles. These are similar to those anticipated for the NIF direct-drive target. For the exploding- 
(a) Exploding foil
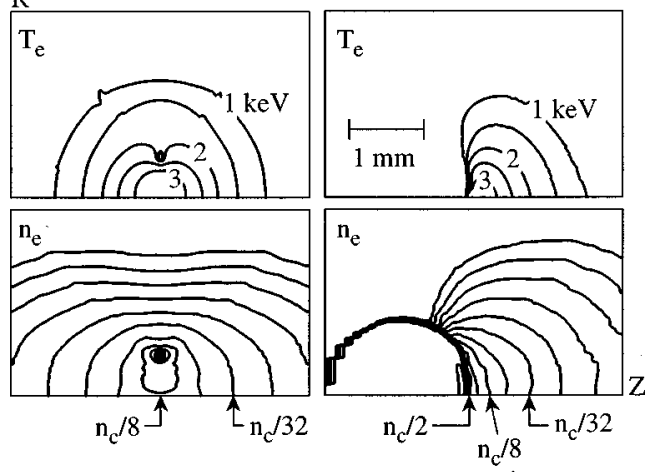

FIG. 4. Contour plots of $T_{e}$ and $n_{e}$ at 2.6 ns simulated with SAGE for (a) an 18- $\mu$ m-thick exploding foil and (b) a solid target (a 2-mm-diam $\mathrm{CH}$ sphere).

foil plasma, the density profile on axis has a full width at half maximum of $1 \mathrm{~mm}$. For the solid target, the scale length $L_{n} \sim 0.5 \mathrm{~mm}$.

One interesting feature seen in the density contour plot of Fig. 4(a) for the exploding foil is a high-density off-axis region, topologically a ring structure. This effect was also found in earlier experiments on the former 24-beam OMEGA system. ${ }^{11}$ It occurs because the centrally peaked primary beams cause a more rapid explosion of the on-axis portion of the foil. The edge of the foil is only weakly heated by the primary beams. The off-axis ring expands because of heating by the obliquely incident secondary beams, resulting in a flow of mass towards the axis. This compensates for what would otherwise be a rapid fall of density with respect to time in the center of the plasma.

The predicted temporal evolution of the temperature and density in the center of an exploding-foil plasma are plotted in Fig. 5. The temperature rises rapidly around $1 \mathrm{~ns}$ when the thermal front penetrates to the center, and it rises again at 2 ns when the secondary beams turn on. It falls rapidly at $3 \mathrm{~ns}$ when the secondary beams switch off. The density, which has fallen to $\sim n_{c} / 5$ at the start of the secondary beams, stays between $n_{c} / 5$ and $n_{c} / 10$ for the next $2 \mathrm{~ns}$ as a result of the flow of mass from the off-axis ring seen in Fig. 4(a). NIFrelevant conditions are thus maintained throughout the time period of the secondary beams $(2-3 \mathrm{~ns})$.

\section{PLASMA CHARACTERIZATION}

The electron temperature of the exploding-foil plasmas was diagnosed using time-resolved x-ray spectroscopy. High- $Z$ microdots, composed of $\mathrm{Ti}$ and $\mathrm{CaF}$ (1000 $\AA$ thick and $200 \mu \mathrm{m}$ in diameter), were embedded in the center of the foil targets. They served as tracer elements to diagnose the electron temperature predicted in Fig. 5(a), using the sensitivity of the $K$-shell emission lines of Ti and $\mathrm{Ca}$ to variations in the electron temperature for temperatures up to the predicted maximum of $\sim 4 \mathrm{keV}$. Exploding-foil plasmas were used for the temperature measurement because the tracer elements diagnosed the center of the plasma. In a solid target, a tracer element embedded at some depth in the target moves a significant distance through the corona during the interac-
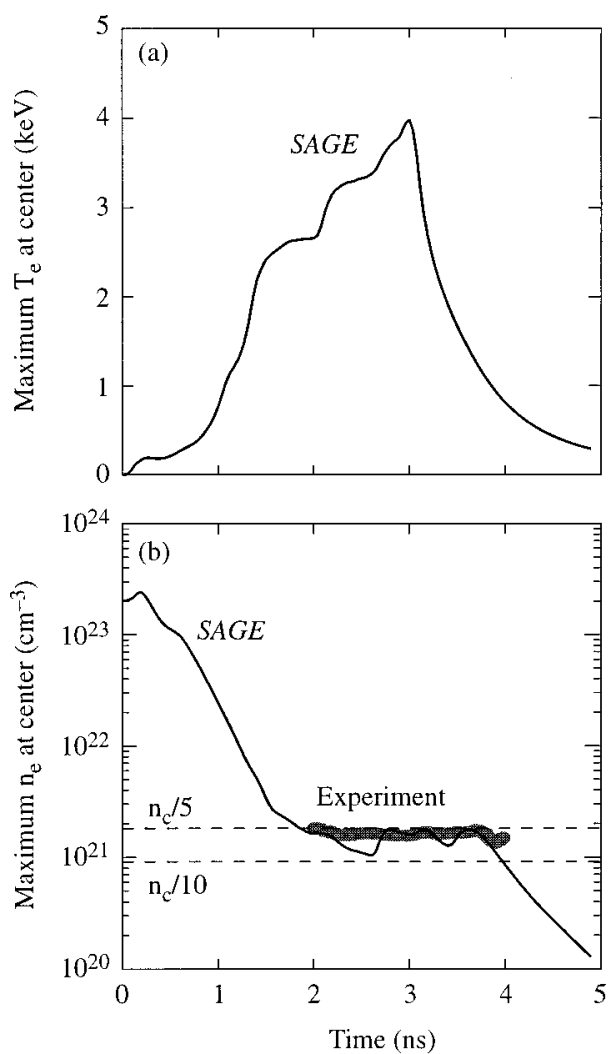

FIG. 5. SAGE predictions for the time dependence of (a) $T_{e}$ and (b) $n_{e}$, at the center of an exploding-foil plasma. This temperature was diagnosed using a tracer layer containing $\mathrm{Ti}$ and $\mathrm{Ca}$ placed in the center of the target. The electron density inferred from the streaked Raman spectrum for a number of shots with different interaction beam timings is also plotted.

tion, encountering a wide range of electron temperatures and densities, and interpretation is more complex. Targets without microdots were investigated to verify that the microdot did not affect the parametric instabilities or plasma hydrodynamics in a significant manner.

Streaked and high-resolution, time-integrated x-ray spectra of $\mathrm{Ti}$ and $\mathrm{Ca}$ tracer elements in exploding-foil plasmas were recorded with flat crystal spectrographs. The timeresolved measurement shown in Fig. 6(a) was photometrically calibrated for spectral sensitivity with the timeintegrated measurement shown in Fig. 6(b). The spectral range was selected to cover the $K$-shell emission of $\mathrm{Ti}$ and $\mathrm{Ca}$. Both instruments had similar views of the plasma. The time-integrated instrument, which was calibrated using measured crystal reflectivities and published film sensitivities, utilized an ADP (ammonium dihydrogen phosphate) or a PET (pentaerythritol) crystal to disperse the spectrum onto Kodak DEF (direct exposure) film. The x-ray streak camera $^{13}$ utilized a RbAP (rubidium acid phthalate) crystal to disperse the spectrum onto a fluffy $\mathrm{KBr}$ photocathode with a 30 ps temporal resolution. The spectra were recorded on Kodak $T$-max 3200 film. The sweep speed of the streak camera was measured using a temporally modulated fiducial pulse. The film from both instruments was digitized using a PDS (Perkin-Elmer Photometric Data Systems) microdensitometer and corrected for film sensitivity. 

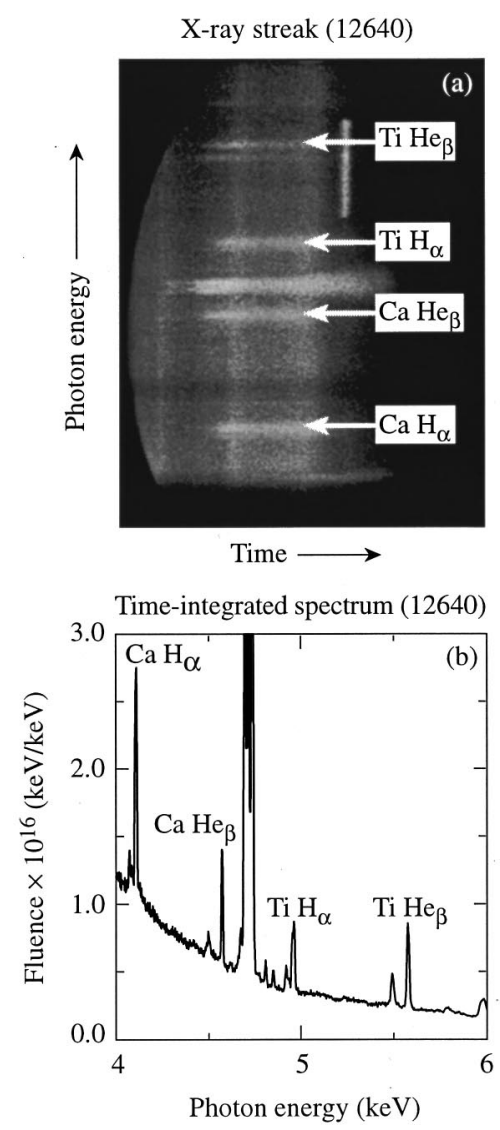

FIG. 6. (a) Streaked x-ray spectra and (b) high-resolution, time-integrated $\mathrm{x}$-ray spectra of $\mathrm{Ti}$ and $\mathrm{Ca}$ in a typical exploding-foil plasma.

The electron temperature was diagnosed using the measured line ratios of hydrogen- and helium-like charge states of $\mathrm{Ca}$ and $\mathrm{Ti}$. In the exploding-foil plasmas the ionization time of the relevant $\mathrm{Ca}$ and $\mathrm{Ti}$ ions is longer than the interaction time $(\sim 1 \mathrm{~ns})$; therefore, a nonsteady-state calculation of the evolution of the line intensities was required. Using the time-dependent temperature and density predicted by SAGE for the target center (see Fig. 5), the evolution of the diagnostic lines $\left(\mathrm{H}_{\alpha}\right.$ and $\mathrm{He}_{\beta}$ of $\mathrm{Ti}$ and $\left.\mathrm{Ca}\right)$ was calculated with the time-dependent FLY atomic physics $\operatorname{code}^{14}$ (based on Ref. 15), which solves the ionic rate equations including ionization, excitation, and recombination (radiative, collisional, and dielectronic). The SAGE/FLY predictions of the Ti and $\mathrm{Ca} \mathrm{H}_{\alpha}: \mathrm{He}_{\beta}$ emission line ratios (shaded region) are plotted in Fig. 7 with the measured line ratios (circle symbols). The shaded region is bounded by the FLY predictions for the SAGE-predicted $T_{e}$ and 1.2 times the SAGEpredicted $T_{e}$. The Ti measurement extends from just below the lower curve at $2 \mathrm{~ns}$ to just below the upper curve at $3 \mathrm{~ns}$, and the $\mathrm{Ca}$ measurement is closer to the upper curve at all
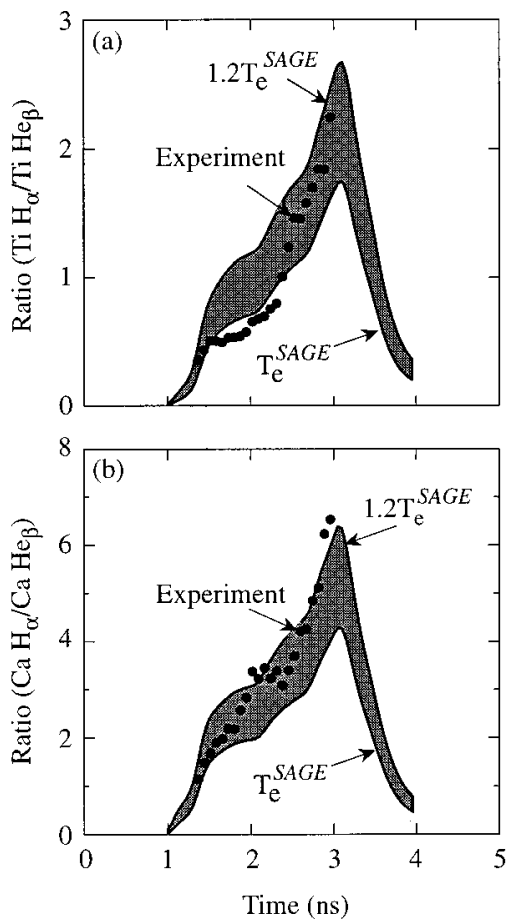

FIG. 7. SAGE/FLY predictions of the $\mathrm{Ti}$ and $\mathrm{Ca}_{\alpha}: \mathrm{He}_{\beta}$ emission line ratios (shaded region) together with the measured line ratios (circle symbols). The shaded region is bounded on the lower side by the FLY prediction using the SAGE-predicted $T_{e}$ and on the upper side by the FLY prediction for 1.2 times the SAGE $T_{e}$. Agreement with SAGE/FLY predictions is found for the measured $\mathrm{Ti}$ and $\mathrm{Ca}$ line ratios, indicating a peak $T_{e}$ of $\sim 4$ $\mathrm{keV}$.

times. Based on this agreement with SAGE/FLY predictions for both line ratios, a peak $T_{e}$ of $\sim 4 \mathrm{keV}$ is inferred. The opacity of these lines due to self-absorption was established experimentally to be insignificant: when the thickness of the microdot was doubled, the measured intensity doubled. The isoelectronic method ${ }^{16}$ for measuring the electron temperature was not available due to uncertainty in the relative amounts of $\mathrm{Ti}$ and $\mathrm{Ca}$.

The peak plasma electron density in the exploding-foil plasmas was diagnosed using the near-backscattered SRS spectrum. ${ }^{4}$ In the center of an exploding foil, SRS is an absolute instability at the peak of the parabolic profile and consequently has an intensity threshold of $7 \times 10^{13} \mathrm{~W} / \mathrm{cm}^{2}$. A narrow spectral emission was observed with a time-resolved spectrograph [see Fig. 8(a)]. The observed SRS wavelength determined from the 50\% intensity point on the longwavelength side of the narrow peak is compared with the wavelength predicted from a SAGE simulation in Fig. 8(b), using the following relationship between the density and the scattered wavelength:

$$
\frac{n}{n_{c}}=\left(1-\frac{\lambda_{0}}{\lambda_{s}}\right)^{2}-3 \frac{v_{T}^{2}}{c^{2}}\left[\frac{\sqrt{\left(1-4 \frac{\lambda_{0}}{\lambda_{s}}+\frac{\lambda_{0}^{2}}{\lambda_{s}^{2}}\right)^{2}-\left(1-12 \frac{v_{T}^{2}}{c^{2}}\right)\left(1-\frac{\lambda_{0}^{2}}{\lambda_{s}^{2}}\right)}-\left(1-4 \frac{\lambda_{0}}{\lambda_{s}}+\frac{\lambda_{0}^{2}}{\lambda_{s}^{2}}\right)}{1-12 \frac{v_{T}^{2}}{c^{2}}}\right]
$$



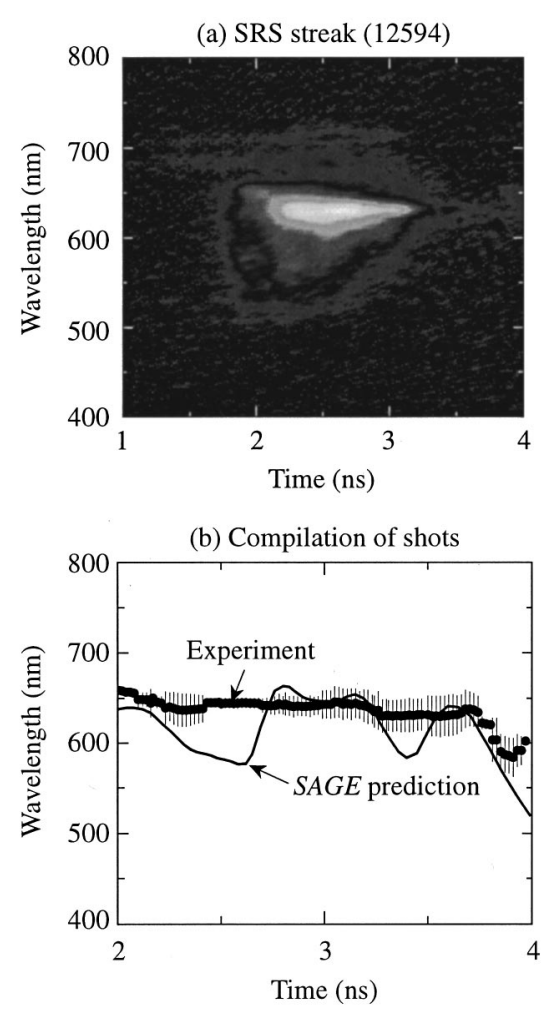

FIG. 8. (a) Measured SRS spectrum of an exploding-foil plasma with the interaction beam timed to start at $2 \mathrm{~ns}$, and (b) measured peak SRS wavelengths compiled from a series of shots with different interaction beam timings compared with SAGE predictions. The vertical lines associated with the experimental curve in (b) represent variations between different shots.

where $v_{T}$ is the electron thermal velocity, $c$ is the speed of light, $\lambda_{0}$ is the wavelength of the incident laser light, and $\lambda_{s}$ is the scattered wavelength. This equation was obtained from the dispersion relations and phase-matching conditions for all the waves involved in the SRS.

The experimental wavelength data in Fig. 8(b) represent a series of shots with different interaction beam timings. The error bars on the experimental data represent the statistical variation in the measured wavelength over the compilation of shots. The experimental data of Fig. 8(b), translated into the inferred electron densities, are shown as an overlay in Fig. 5(b). From both figures the agreement between experiment and simulation is seen to be close, and the predicted onset of the drop in density around $3.7 \mathrm{~ns}$ is observed. The oscillations predicted by SAGE are associated with the offaxis mass converging onto the axis in a geometry with cylindrical symmetry. In the experiment the azimuthal asymmetries associated with the finite number of beams in each ring are expected to result in this behavior being averaged out. Both simulation and experiment are consistent with the production of a plasma with the peak $n_{e}$ staying between $0.1 n_{c}$ and $0.2 n_{c}$ for approximately $2 \mathrm{~ns}$.

\section{LASER-PLASMA INTERACTION EXPERIMENTS}

Signal levels of the direct backscatter of SRS and SBS from the interaction beam were recorded with the experimental setup shown in Fig. 9. The backscattered SRS and SBS signals were measured through the focusing lens with a full-

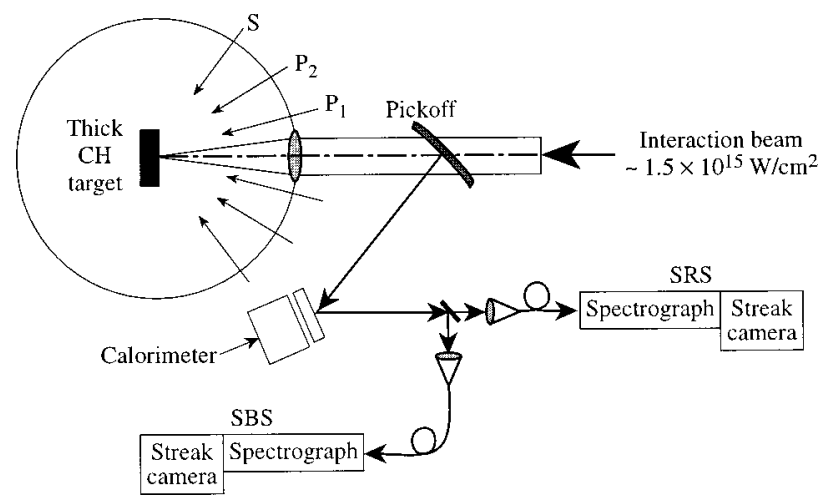

FIG. 9. Experimental setup used to measure the SRS and SBS transmitted back through the focusing lens. The calorimeter measured the sum of the SRS and SBS energies reflected off the pickoff. Significant SRS reflections were made off each of the pickoff surfaces.

aperture pickoff whose front surface was uncoated and whose back surface was AR (sol gel) coated for $351 \mathrm{~nm}$. The back-surface reflectivity in the 400 to $800 \mathrm{~nm}$ range lies between $2 \%$ and $3 \%$ and is close to the reflectivity of the front surface. This unfortunately degrades the temporal resolution of the SRS measurements to $\sim 250$ ps and lengthens the SRS signals by the same amount.

A small fraction of the backscattered light was directed via optical fibers to two visible spectrographs, one near 351 $\mathrm{nm}$ to look for SBS and one covering the 400-700 $\mathrm{nm}$ range to look for SRS. Both spectrographs were outfitted with streak cameras. The remainder of the backscattered light was measured with an unfiltered calorimeter (which does not distinguish between SBS and SRS). This calorimeter resolved a small fraction of a Joule of light backscattered from the target.

The SRS measurement used a 1/3 m Czerny-Turner spectrograph with $8 \mathrm{~nm}$ spectral resolution coupled to a streak camera. A $20 \mathrm{~m}, 50 \mu \mathrm{m}$ gradient index fiber transmitted the SRS light from a pickoff at the backscatter station to the entrance slit of the spectrograph. The broad bandwidth of the SRS light necessitated a wavelength-dependent group velocity dispersion correction which amounted to $\sim 5$ ns between 351 and $700 \mathrm{~nm}$. This correction was verified by measuring the time delay between the second-order $351 \mathrm{~nm}$ signal and a $700 \mathrm{~nm}$ SRS/TPD signal. Any error associated with this correction is estimated to be $<50$ ps over this spectral range and consequently negligible. It was found that there was no measurable contribution to the streaked SRS spectra from the secondary beams.

The SBS measurement used a $1 \mathrm{~m}$ Czerny-Turner spectrograph with $\sim 0.05 \mathrm{~nm}$ spectral resolution, coupled to a streak camera with $\sim 50$ ps temporal resolution. The background light level entering this spectrograph was measured with shots without the interaction beam. For exploding-foil plasmas, the time-resolved backscatter spectra were dominated by scattered light from the primary and secondary beams and no SBS feature could be identified. Shots with and without the interaction beam at $1.5 \times 10^{15} \mathrm{~W} / \mathrm{cm}^{2}$ were barely distinguishable. (This was in contrast to earlier experiments carried out without a DPP in the interaction beam, 


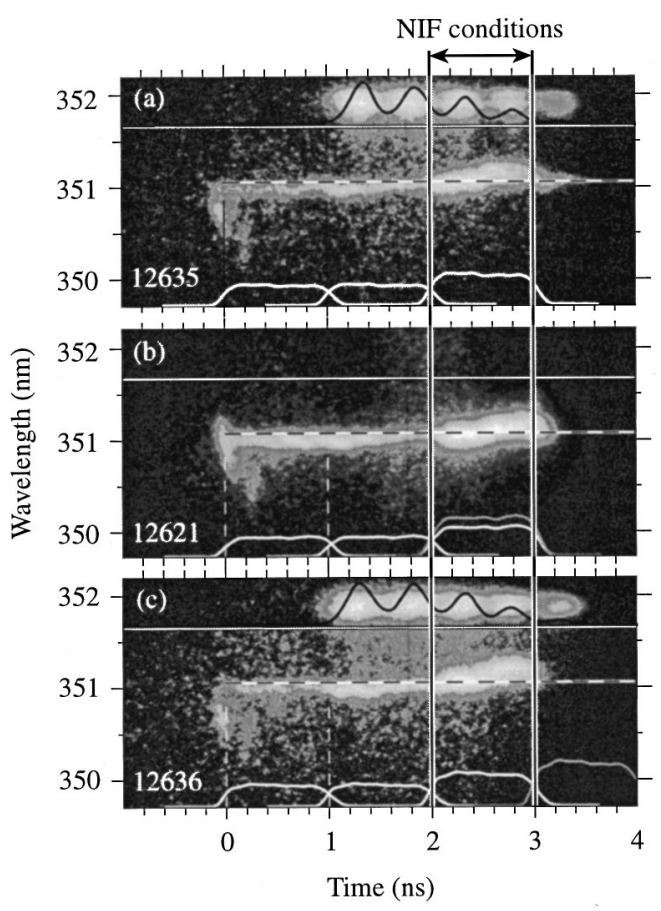

FIG. 10. Streaked spectra around $351 \mathrm{~nm}$ of light backscattered from exploding-foil plasmas through the interaction-beam focus lens for (a) no interaction beam, (b) interaction beam at $2 \mathrm{~ns}$, and (c) interaction beam at 3 ns. A multipulse timing fiducial [shown at the top of (a) and (c) with the solid line indicating its temporal shape] enabled an absolute time origin to be assigned to the backscattered light. The measured streak records of the four groups of laser beams are also shown. The horizontal dashed line indicates the unshifted laser wavelength.

where the SBS reflectivity exceeded $10 \% .^{17}$ ) The energy response of the SBS spectrograph was estimated using shots taken without the interaction beam. In this case, there was no scattered light in the SRS range, so the energy recorded in the calorimeter corresponded to the signal in the SBS spectrograph.

Measured backscatter SBS spectra are presented in Fig. 10 for the exploding-foil plasmas after the peak density has dropped to $\sim n_{c} / 5$. The time during which NIF temperatures and scale lengths are achieved extends from 2 to 3 ns. Figure 10(a) shows the temporal evolution of the spectrum near 351 $\mathrm{nm}$ when the interaction beam was not fired and was used to establish the level of background light. The interaction beam was fired at $2 \mathrm{~ns}$ in Fig. 10(b) and at $3 \mathrm{~ns}$ in Fig. 10(c). Since there are no significant differences between the three images, it is clear that no measurable contribution from the interaction beam to the signal near $351 \mathrm{~nm}$ was observed for either timing. The energy response of the spectrograph places an upper limit on the SBS reflectivity of $0.1 \%$ for these experiments.

A similar set of streaked spectra is shown in Fig. 11 for solid-target plasmas. The signal near $t=0$ in all three cases is interpreted as light reflected from the critical surface with a Doppler blueshift from the expanding plasma. This is consistent with the SAGE prediction for the time-dependent absorbed power [Fig. 3(b)]. Less signal is seen during the second set of primary beams, as they were incident at greater angles from the collection lens. The feature around $351 \mathrm{~nm}$

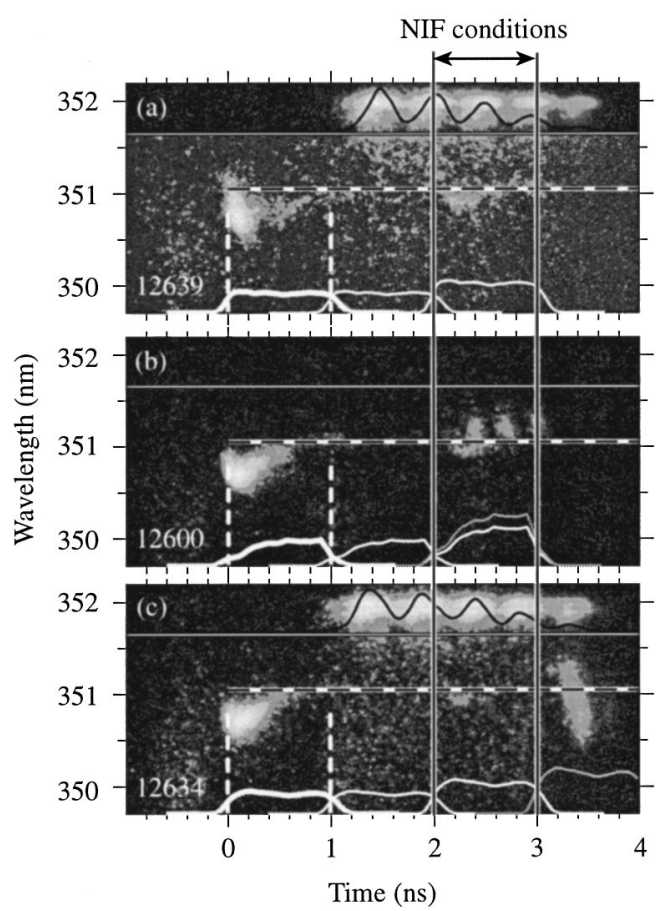

FIG. 11. Same as Fig. 10 but for the solid targets (partial spheres of $2 \mathrm{~mm}$ diameter).

in Fig. 11(b) between 2 and 3 ns corresponds to a $0.1 \%$ reflection of the interaction beam from near the criticaldensity surface. The total energy recorded by the spectrograph was $3.1 \mathrm{~J}$, corresponding to $0.7 \%$ of the interaction beam energy, so most of the energy was in the scattered $P_{1}$ beams. The feature after $3 \mathrm{~ns}$ in Fig. 11(c) is from what would, in the absence of the interaction beam, be a rapidly cooling plasma. This plasma, which still has a criticaldensity surface, is strongly absorbing (close to 100\% absorption is predicted) and is locally heated by the interaction beam. The observed backscatter is consistent with a backscattered SBS energy fraction of $0.2 \%$. Thus, no significant SBS was observed in either the exploding-foil or solid-target interaction experiments under NIF direct-drive conditions.

Small amounts of SRS were observed in both types of plasma. For the exploding-foil plasmas, SRS from the electron-density maximum was used as a density diagnostic (see preceding section). For solid-target plasmas, timeresolved SRS was measured through the focusing lens (direct backscatter) and at $20^{\circ}$ from the backscatter direction (Fig. 12). Figures 12(a) and 12(b) show the temporal evolution of the SRS backscatter spectra through the lens, with the interaction beam fired at 2 and $2.5 \mathrm{~ns}$, respectively, with peak intensity $1.5 \times 10^{15} \mathrm{~W} / \mathrm{cm}^{2}$. Both images exhibit broad spectral features coincident in time with the interaction beam and extending from 420 to $540 \mathrm{~nm}$. The weak feature at $700 \mathrm{~nm}$ appears to be $\omega_{0} / 2$ light from the two-plasmon decay instability driven by the primary and secondary beams.

An upper limit on the backscattered SRS energy can be estimated from the calorimetry and the SBS signal. As mentioned above, the spectral energy response of the SBS spectrograph was determined on shots where the interaction beam was not fired. By subtracting the SBS energy in the 


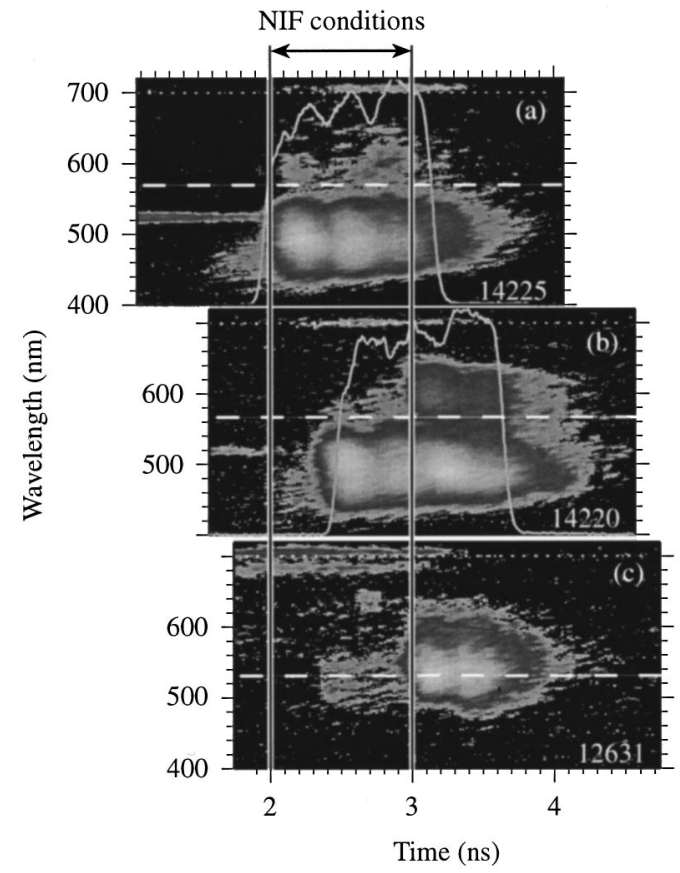

FIG. 12. Streaked SRS spectra from solid-target plasmas: (a) and (b) directly back through the lens, with SSD on $(0.25 \mathrm{THz}$ UV bandwidth), and (c) at $20^{\circ}$ to the lens with SSD off. The interaction beam was timed at $2 \mathrm{~ns}$ in (a) and at $2.5 \mathrm{~ns}$ in (b) and (c). The temporal shape of the interaction beam is shown as an overlay in (a) and (b). The targets were flat in (a) and (b) and a partial sphere of $2 \mathrm{~mm}$ diameter in (c).

spectrograph from the total energy recorded in the calorimeter, the energy in the SRS signal can in principle be estimated. However, due to the shot-to-shot fluctuations in the signal levels there is a large uncertainty associated with this subtraction. In addition, the final turning mirror has $\sim 100 \%$ reflectivity at $351 \mathrm{~nm}$ but a flat $\sim 12 \%$ reflectivity between 400 and $700 \mathrm{~nm}$, further reducing the SRS contribution in the calorimeter. Taking all these factors into account, the residual SRS energy in the calorimeter is $0 \mathrm{~J}$ with an uncertainty of $25 \mathrm{~J}$. The upper limit of $25 \mathrm{~J}$ corresponds to a maximum SRS backscattered energy fraction of 5\%, but clearly a lower value is more likely. An accurate measurement of the SRS fraction will require further experiments.

Landau damping is very strong in this densitytemperature regime $\left(k \lambda_{D} \approx 0.5\right.$ at $570 \mathrm{~nm}$ during the hightemperature portion of the interaction), which suggests that the observed SRS originates in filaments where Landau damping is strongly reduced. ${ }^{18}$

A time-resolved sidescattered SRS spectrum from a solid target, at $20^{\circ}$ from the interaction beam direction (the normal to the original target surface), is shown in Fig. 12(c) with the interaction beam fired at $2.5 \mathrm{~ns}$. No SRS is observed in sidescattering during the hot NIF-like plasma phase between $t=2$ and $3 \mathrm{~ns}$, but sidescattering sets in abruptly when the secondary beams turn off at $t=3 \mathrm{~ns}$. At this time the background plasma cools down, although this alone may not explain the abrupt onset of sidescattering. The sidescatter SRS appears at longer wavelengths (i.e., higher densities) than the filamentary SRS seen in backscattering between 2 and $3 \mathrm{~ns}$. This is consistent with the onset of SRS at longer wavelengths in direct backscattering at $3 \mathrm{~ns}$ [Fig. 12(b)].

It is possible that some SRS was present just outside the $f / 6$ cone of the interaction-beam focusing lens. However, this is unlikely to be significant, based on the lack of SRS at $20^{\circ}$ from 2 to $3 \mathrm{~ns}$.

Combining the backscatter and sidescatter SRS observations it is reasonable to conclude that the directionality of the filamentary (low-density) SRS signal is due to the "horn antenna" effect, which directs the incident $351 \mathrm{~nm}$ light into the filaments and then funnels the backscattered SRS signal through the same horn to the focusing lens. Within the filament the SRS signal is guided by the filament as well as by the SRS gain, while in the region of the horn geometrical optics is applicable and the backscattered SRS light is guided by the density structure making up the horn. It is impossible to determine the exact background density within which the filaments are created since the SRS wavelength merely reflects the density within the filaments. It is clear that the 2D-SSD bandwidth used in this experiment $(0.25 \mathrm{THz}$ in the UV) is insufficient to suppress the filamentation.

The density gradient threshold for Raman scattering (e.g., that given by Kruer ${ }^{4}$ ) yields $I \sim 1.8 \times 10^{15} \mathrm{~W} / \mathrm{cm}^{2}$, and SRS would be expected to originate within the speckles produced by the phase plates. During the hot NIF-like plasma phase, however, Landau damping is strong enough to suppress this SRS completely while in the cooling phase (past $t=3 \mathrm{~ns})$ SRS can occur and is seen between 0.1 and $0.2 n_{c}$. This type of Raman scattering (without filamentation) is still preferentially directed in the backward direction, but it is much less collimated, which thus allows its observation through the lens as well as at $20^{\circ}$ away from the backscatter direction.

\section{CONCLUSION}

Long-scale-length plasmas, with parameters relevant to the peak of direct-drive NIF laser pulses, have been produced and characterized on the OMEGA laser system. Temperatures of $\sim 4 \mathrm{keV}$ and densities of $\sim 0.2 n_{c}$ have been measured in agreement with hydrocode predictions and are consistent with density scale lengths of $\sim 0.5-1.0 \mathrm{~mm}$. Experiments have shown that these plasmas have an SBS reflectivity of less than $0.1 \%$ and an SRS reflectivity of less than $\sim 5 \%$, even when the interaction beam intensity is 1.5 $\times 10^{15} \mathrm{~W} / \mathrm{cm}^{2}$, eight times higher than the NIF cluster intensity. The measured backscattered SRS signals are believed to originate in filaments at $n_{e}<0.1 n_{c}$, as otherwise they would be suppressed by the strong Landau damping that occurs at the measured temperatures. The threshold intensity for SRS was not determined since experiments were not performed with a lower-intensity interaction beam. The calculated SRS and SBS intensity thresholds were comparable to the intensity of the interaction beam at $1.5 \times 10^{15} \mathrm{~W} / \mathrm{cm}^{2}$; however, only SRS was observed in the experiment.

On the basis of these experiments, it is concluded that SRS and SBS are not likely to present a problem for the coronal plasmas at the peak of the NIF direct-drive laser pulse. Future experiments on OMEGA will vary the intensity 
of the interaction beam and will address the parametric instabilities of the coronal plasmas in the foot and transition regions of the NIF direct-drive laser pulse.

\section{ACKNOWLEDGMENTS}

The authors thank Dr. C. P. Verdon for providing the predicted NIF profiles and T. Kessler and D. Smith for the design and fabrication of the DPPs used in these experiments. The authors also thank the referee for a very thorough reading of the first manuscript and many helpful suggestions. This work was supported by the U.S. Department of Energy (DOE) Office of Inertial Confinement Fusion under Cooperative Agreement No. DE-FC03-92SF19460, the University of Rochester, and the New York State Energy Research and Development Authority. The support of DOE does not constitute an endorsement by DOE of the views expressed in this article.

${ }^{1}$ C. P. Verdon, Bull. Am. Phys. Soc. 38, 2010 (1993).

${ }^{2}$ S. E. Bodner, D. G. Colombant, J. H. Gardner, R. H. Lehmberg, S. P. Obenschain, L. Phillips, A. J. Schmitt, J. D. Sethian, R. L. McCrory, W. Seka, C. P. Verdon, J. P. Knauer, B. B. Afeyan, and H. T. Powell, Phys. Plasmas 5, 1901 (1998).

${ }^{3}$ J. D. Lindl, Phys. Plasmas 2, 3933 (1995).

${ }^{4}$ W. L. Kruer, in The Physics of Laser Plasma Interactions, Frontiers in Physics, Vol. 73, edited by D. Pines (Addison-Wesley, Redwood City, CA, 1988).

${ }^{5}$ J. C. Fernández, B. S. Bauer, J. A. Cobble, D. F. DuBois, G. A. Kyrala, D. S. Montgomery, H. A. Rose, H. X. Vu, R. G. Watt, B. H. Wilde, M. D. Wilke, W. M. Wood, B. H. Failor, R. Kirkwood, and B. J. MacGowan, Phys. Plasmas 4, 1849 (1997).

${ }^{6}$ B. J. MacGowan, B. B. Afeyan, C. A. Back, R. L. Berger, G. Bonnaud, M.
Casanova, B. I. Cohen, D. E. Desenne, D. F. DuBois, A. G. Dulieu, K. G. Estabrook, J. C. Fernandez, S. H. Glenzer, D. E. Hinkel, T. B. Kaiser, D. H. Kalantar, B. I. Kauffman, R. K. Kirkwood, W. L. Kruer, A. B. Langdon, B. F. Lasinski, D. S. Montgomery, J. D. Moody, D. H. Munro, L. V. Powers, H. A. Rose, C. Rousseaux, R. E. Turner, B. H. Wilde, S. C. Wilks, and E. A. Williams, Phys. Plasmas 3, 2029 (1996).

${ }^{7}$ T. R. Boehly, D. L. Brown, R. S. Craxton, R. L. Keck, J. P. Knauer, J. H. Kelly, T. J. Kessler, S. A. Kumpan, S. J. Loucks, S. A. Letzring, F. J. Marshall, R. L. McCrory, S. F. B. Morse, W. Seka, J. M. Soures, and C. P. Verdon, Opt. Commun. 133, 495 (1997).

${ }^{8}$ Y. Lin, T. J. Kessler, and G. N. Lawrence, Opt. Lett. 21, 1703 (1996).

${ }^{9}$ R. S. Craxton and R. L. McCrory, J. Appl. Phys. 56, 108 (1984).

${ }^{10}$ M. C. Richardson, P. W. McKenty, F. J. Marshall, C. P. Verdon, J. M. Soures, R. L. McCrory, O. Barnouin, R. S. Craxton, J. Delettrez, R. L. Hutchison, P. A. Jaanimagi, R. Keck, T. Kessler, H. Kim, S. A. Letzring, D. M. Roback, W. Seka, S. Skupsky, B. Yaakobi, S. M. Lane, and S. Prussin, in Laser Interaction and Related Plasma Phenomena, edited by H. Hora and G. H. Miley (Plenum, New York, 1986), Vol. 7, pp. 421448.

${ }^{11}$ W. Seka, R. S. Craxton, R. E. Bahr, D. L. Brown, D. K. Bradley, P. A. Jaanimagi, B. Yaakobi, and R. Epstein, Phys. Fluids B 4, 432 (1992).

${ }^{12}$ S. Skupsky, R. W. Short, T. Kessler, R. S. Craxton, S. Letzring, and J. M. Soures, J. Appl. Phys. 66, 3456 (1989).

${ }^{13}$ D. H. Kalantar, P. M. Bell, R. L. Costa, B. A. Hammel, O. L. Landen, T. J. Orzechowski, J. D. Hares, and A. K. L. Dymoke-Bradshaw, in 22nd International Congress on High-Speed Photography and Photonics, edited by D. L. Paisley and A. M. Frank (SPIE, Bellingham, WA, 1997), Vol. 2869, pp. 680-685.

${ }^{14}$ Available from Cascade Applied Sciences, Inc., P.O. Box 4477, Boulder, CO 80306.

${ }^{15}$ R. W. Lee, B. L. Whitten, and R. E. Stout, J. Quant. Spectrosc. Radiat. Transf. 32, 91 (1984).

${ }^{16}$ R. S. Marjoribanks, M. C. Richardson, P. A. Jaanimagi, and R. Epstein, Phys. Rev. A 46, R1747 (1992).

${ }^{17}$ A. V. Chirokikh, R. S. Craxton, D. D. Meyerhofer, A. Simon, W. Seka, and R. P. Drake, Bull. Am. Phys. Soc. 42, 1882 (1997).

${ }^{18}$ R. W. Short and A. Simon, Phys. Plasmas 5, 4134 (1998). 